\title{
Reactivación de la consulta y cirugía electiva durante la pandemia: recomendaciones de seguridad. Medicina Basada en Evidencia
}

\author{
Reactivation of the consultation and elective surgery during the \\ pandemic: Safety recommendations. Evidence Based Medicine \\ Dr. Jesús Cuenca-Pardo, ${ }^{*}$ Dra. Estela Vélez-Benítez, ${ }^{\ddagger}$ Dr. Martín Morales-Olivera, ${ }^{\ddagger}$ \\ Dr. Rufino Iribarren-Moreno,${ }^{\ddagger}$ Dra. Livia Contreras-Bulnes, ${ }^{\S}$ Dr. Javier Bucio-Duarte ${ }^{\ddagger}$
}

Palabras clave: SARS-CoV-2, COVID-19, pandemia por coronavirus, factores de riesgo, diagnóstico, pruebas diagnósticas, procedimientos quirúrgicos, cuidados preventivos, medidas de protección personal.

\section{RESUMEN}

Realizamos una revisión sistemática sobre COVID 19 y la reactivación de los procedimientos electivos y buscamos la mejor evidencia médica disponible. Con los datos obtenidos, elaboramos recomendaciones preventivas para el personal de salud y para los pacientes; de la misma manera elaboramos una infografía como una guía rápida, sencilla, con la información suficiente, que permita al cirujano tomar las mejores decisiones. El diagnóstico de la enfermedad se realiza a través de un cuestionario, PCR, pruebas inmunológicas, detección de antígenos del virus y tomografía simple de tórax. La mayor sensibilidad se alcanza al combinar las pruebas. El personal de salud deberá realizarse pruebas diagnósticas y el personal infectado o de alto riesgo no deberá atender pacientes. Todos los pacientes se deben considerar sospechosos y se les debe realizar pruebas diagnósticas. Los casos positivos podrán operarse hasta que se recuperen de la enfermedad. La estratificación de riesgo de contagio es importante para la toma de decisiones y la protección que se deberá utilizar. La pandemia por COVID-19 continuará por un largo tiempo; el retorno a las actividades habituales se está realizando con fines económicos y no porque la pandemia se haya controlado. Para disminuir el riesgo de contagio es necesario utilizar las guías emitidas por diferentes organizaciones y las recomendaciones indicadas por las autoridades sanitarias. Las recomendaciones que hacemos en este trabajo no son obligatorias, ni establecen un nuevo estándar de atención. Los pacientes de cirugía estética deberán ser atendidos en áreas «libres de COVID» y no exponerlos a un contagio.

\section{ABSTRACT}

We carried out a systematic review on COVID 19 and the reactivation of elective procedures and searched for the best medical evidence available. With the data obtained, we prepared preventive recommendations for health personnel and patients; in the same way, we created an infographic as a quick, simple guide, but with enough information to allow the surgeons to make the best decisions. The diagnosis of the disease is made through a questionnaire, $P C R$, immunological tests, detection of virus antigens and a simple chest tomography. The highest sensitivity is achieved by combining the tests. Health personnel should undergo diagnostic tests and those infected or high-risk personnel should not attend patients. All patients should be considered suspicious and diagnostic tests should be performed and positive cases may be operated on until they recover from the disease. Contagion risk stratification is important for decision making and the protection that should be used. The COVID-19 pandemic will continue for a long time and the return to everyday activities is being carried out for economic purposes and not because the pandemic has been controlled to reduce the risk of contagion, it is necessary to use the guidelines issued by different organizations and the recommendations indicated by health authorities. The recommendations that we make in this work are not mandatory, nor do they establish a new standard of care. Cosmetic surgery patients should be treated in «COVID free» areas and must not be exposed to contagion.

nocida en la ciudad de Wuhan, provincia de Hubei, China. Tiempo después, se demostró que la enfermedad era producida por un nuevo coronavirus (2019-nCoV), que posteriormente 
fue denominado como síndrome respiratorio agudo severo coronavirus 2 (SARS-CoV-2). ${ }^{1,2}$ La Organización Mundial de la Salud (OMS) lo nombró COVID-19.3,4 La mayoría de los coronavirus son zoonóticos transmitidos a los humanos a través del contacto con animales infectados; los murciélagos y las serpientes son el reservorio natural de la mayoría de los coronavirus. ${ }^{1}$ El COVID-19 es un nuevo coronavirus beta con agente de transmisión primaria desconocida; la transmisión secundaria se realiza de persona a persona por contacto o inhalación de gotitas respiratorias que contienen el virus. ${ }^{2,5}$ El 31 de enero de 2020, la OMS declaró la epidemia de COVID-19 como «emergencia de salud pública de importancia internacional». Al reportarse casos en la mayoría de los países, fue calificado de pandemia el 11 de marzo, con una mortalidad del 2 al 3\%, ${ }^{6,7}$ siendo una emergencia de salud pública internacional con un alto riesgo para los países con sistemas de salud vulnerables. ${ }^{7}$

Los servicios de salud deben estar preparados para la emergencia actual que se propaga rápidamente y cambia exponencialmente en cualquier momento. ${ }^{8,9}$ Es necesario establecer un protocolo definido y disponible para los profesionales de la salud que atienden pacientes durante la contingencia y es clave para minimizar la propagación de COVID-19. ${ }^{10}$

Estudios previos de epidemias ocasionadas por coronavirus sugieren que la recuperación de COVID-19 podría conferir inmunidad contra la reinfección, al menos temporalmente. Sin embargo, la respuesta inmune al COVID-19 aún no se comprende completamente y faltan datos definitivos sobre la inmunidad postinfección. En medio de la incertidumbre de esta crisis de salud pública, la ciencia reflexiva y rigurosa será esencial para informar las políticas, la planificación y la práctica de la salud pública. Comprender si la recuperación de COVID-19 confiere inmunidad o si disminuye la gravedad de la reinfección es necesario para poder planificar en forma segura las actividades de una población. La comprensión de la inmunidad post-infección también tiene implicaciones importantes para las evaluaciones epidemiológicas. ${ }^{8-11}$

Es imprescindible entender que el término cirugía «electiva» no significa cirugía opcional y más bien implica que un procedimiento no se indica inmediatamente en respuesta a una emergencia potencialmente mortal, o que compromete la función de una extremidad. Una estimación actual sugiere que más del $50 \%$ de los casos de todas las cirugías electivas, tienen el potencial de ocasionar un daño significativo en los pacientes si son canceladas o retrasadas. La condición fisiológica de una cohorte vulnerable de pacientes puede empeorar rápidamente en ausencia de un cuidado quirúrgico apropiado y la disminución resultante en la salud de los pacientes probablemente los haga más vulnerables a una infección por coronavirus. Los procedimientos electivos se pueden estratificar pragmáticamente en «esenciales», lo que implica que existe un mayor riesgo de resultados adversos al retrasar la atención quirúrgica para un indeterminado periodo de tiempo, versus «no esenciales» $\mathrm{o}$ «discrecionales», que alude a procedimientos que no son sensibles al tiempo por razones médicas, como son los procedimientos estéticos que pueden posponerse. ${ }^{12,13}$

La comprensión actual de la transmisión SARS-CoV-2 se basa en la extrapolación de la experiencia pasada con el brote de SARS-CoV en 2003. ${ }^{14-16}$ Los posibles modos de transmisión incluyen gotitas respiratorias, contacto, fómites y la ruta fecal-oral. Existe evidencia de transmisión aérea del SARS CoV-2 y puede transmitirse a través de procedimientos de generación de aerosoles, como la intubación traqueal. Como con la mayoría de los virus respiratorios, se considera más contagioso cuando el paciente es sintomático, existe la posibilidad de que el SARS-CoV-2 sea transmitido por individuos asintomáticos. Los datos obtenidos al momento sugieren que el SARS-CoV-2 es muy contagioso y que puede llegar a afectar hasta el $80 \%$ de la población mundial. ${ }^{14-23}$

Objetivo. Las autoridades sanitarias en varios países prohibieron la apertura de consultorios médicos y la realización de procedimientos electivos durante la pandemia de COVID-19. La enfermedad permanecerá durante mucho tiempo; sin embargo, la cuarentena pronto terminará y regresaremos a nuestras actividades a pesar del riesgo. Debemos preparar el retorno a la actividad y hacerlo en una forma responsable y segura; algunas prácticas sin los cuidados correspondientes aumentarán el riesgo de contagio. El objetivo del presente 
trabajo es elaborar recomendaciones de seguridad basados en la mejor evidencia médica disponible con la finalidad de reducir el riesgo de contagio para nuestros pacientes y nuestro equipo quirúrgico.

Metodología. Realizamos una revisión sistemática en español e inglés en los sitios de información: PubMed, Embase, Cochrane, Medline, Fisterra, Medigraphic y Google Académico. Las palabras clave en español que utilizamos fueron: SARS-CoV-2, COVID-19 o pandemia por coronavirus, factores de riesgo, diagnóstico, pruebas diagnósticas, procedimientos quirúrgicos, cuidados preventivos, medidas de protección personal. Elaboramos preguntas básicas relacionadas con el tema; buscamos la mejor evidencia médica disponible para contestar cada pregunta y realizamos un consenso de un grupo de cirujanos con experiencia en el tema. Con los datos obtenidos, elaboramos recomendaciones preventivas para el personal de salud y para los pacientes; también elaboramos una infografía como una guía rápida y sencilla, pero con la información suficiente que permita al cirujano realizar sus actividades laborales con mínimo riesgo de contagio.

\section{DIAGNÓSTICO DE COVID-19. SENSIBILIDAD DE LAS PRUEBAS}

Cualquier persona debe considerarse enferma de COVID-19 hasta que se descarte lo contrario mediante los recursos diagnósticos existentes. ${ }^{24-27}$ Actualmente, no existe un método de detección que alcance una sensibilidad y especificidad del $100 \%$; además, en muchos países hay poca disponibilidad de pruebas diagnósticas. Es por ello que como alternativa se utiliza la tomografía computarizada simple de tórax (TC). ${ }^{28}$ La OMS no aconseja las pruebas rápidas para la detección del COVID-19, ya que tienen una baja eficacia, calculan que más del $50 \%$ de los casos con la enfermedad podrían pasar desapercibidos con dichas pruebas. ${ }^{29} \mathrm{El}$ diagnóstico específico se realiza mediante la demostración del virus en pruebas moleculares específicas de muestras respiratorias obtenidas de esputo o lavado bronquial. ${ }^{2,6}$

Diagnóstico por cuadro clínico. Existen varios cuestionarios para la detección del CO-
VID-19, algunos de ellos con acceso electrónico (Figura 1). ${ }^{30,31}$ Los síntomas más comúnmente identificados son fiebre, tos seca, mialgias, ataque al estado general $y$, en su forma grave, cuadros de neumonía y disnea. Menos comunes son la cefalea, diarrea, conjuntivitis, dolor de garganta, hemoptisis, congestión nasal, tos productiva, anosmia y ageusia, erupciones cutáneas y acrocianosis. La forma asintomática o leve se presenta hasta en un $80-90 \%$ de los casos, la forma grave en un $10 \%$ y la condición crítica en un 5\%. Existen dos tipos de pacientes asintomáticos: los que nunca desarrollarán síntomas y los asintomáticos que sí lo harán; los primeros representan una evidencia fuerte para el desarrollo de una inmunidad duradera. Los grupos más susceptibles son los adultos mayores y aquellos con enfermedades como hipertensión arterial, patología cardiopulmonar, cáncer, diabetes, cirrosis, Parkinson o estados de inmunosupresión. 2,6,28,29,32 Las guías clínicas mencionan que si un paciente con síntomas de fiebre, disminución del recuento leucocitario y linfocítico, así como infiltrados pulmonares, que no mejora después de tres días de tratamiento antibiótico, deberá ser sometido a una prueba de diagnóstico de COVID-19 por laboratorio. Cabe mencionar que cualquier paciente con evidencia de infección respiratoria debe asumirse como potencialmente infectado con SARS-CoV-2.2,6

Diagnóstico por laboratorio. Se clasifican en tres grupos, las opciones disponibles dependen de las estrategias sanitarias de cada país. $2,6,33-36$

1. Pruebas rutinarias de laboratorio. Los hallazgos de laboratorio generalmente no son específicos; el recuento de glóbulos blancos suele ser normal o bajo. La linfopenia se asocia con enfermedad grave. El recuento de plaquetas suele ser normal o levemente bajo. La proteína $\mathrm{C}$ reactiva y la velocidad de sedimentación globular están elevadas y la procalcitonina normales. Cuando la procalcitonina está elevada indica una coinfección bacteriana. La ALT/AST, el tiempo de protrombina, creatinina, dímero D, CPK y LDH, al elevarse se asocian con enfermedad grave; la ferritina demuestra la severidad de la enfermedad. ${ }^{2,6}$

2. Pruebas de detección del material genético. Se usa la técnica de PCR (Polymerase 
Chain Reaction, reacción en cadena de la polimerasa), considerada el estándar de oro para infecciones virales similares, como el SARS. La sensibilidad (detección de casos con la enfermedad) de esta prueba va de 40 al 90\%; las tomas nasofaríngeas tienen una sensibilidad menor al $40 \%$, se vuelven más sensibles cuando se toman del tracto respiratorio más bajo, las obtenidas de un lavado bronquial tienen una sensibilidad mayor del 90. También dependen de la etapa del proceso infeccioso. La presencia de los virus disminuye rápidamente durante la primera semana de enfermedad leve; se desconoce la duración absoluta en la que un paciente pueda eliminar el virus; requiere de personal altamente especializado, ya que un procesamiento no adecuado del material genómico puede conducir a resultados erróneos. La especificidad (detección de casos sin la enfermedad) es cercana al $100 \%$. En un paciente positivo, la toma faríngea secundaria puede ser negativa; sin embargo, una siguiente toma puede resultar positiva. Esto no significa una reactivación de la infección. 4,37-43

3. Detección del virus como entidad individual o detección de antígenos. También llamadas pruebas rápidas de detección de antígenos (RADTs, rapid antigen detection tests), son una forma de detección de distintas proteínas estructurales del SARS-CoV-2 que se basa en tiras reactivas que al entrar en contacto con la sustancia diana a detectar, conducen a un cambio generalmente visual (cambio de color). Las ventajas son: rapidez (5-15 minutos entre la toma y la lectura), costo accesible, alcance masivo y no requiere de una técnica compleja ni de personal especializado. Las desventajas son su baja sensibilidad, ${ }^{37}$ con una alta posibilidad de falsos negativos, por lo que en la actualidad este tipo de pruebas no están aprobadas como primera elección diagnóstica. ${ }^{41-47}$

4. Detección de anticuerpos del organismo huésped infectado: prueba serológica. Estas pruebas identifican la presencia de anticuerpos IgM e IgG específicos contra el virus SARS-CoV-2; se requiere de una muestra de sangre, suero o plasma obtenida de la persona estudiada. Su identificación nos orienta sobre las etapas de la enfermedad; la IgM representa el proceso de infección aguda y la IgG indica infección previa o como una respuesta de infecciones secundarias. El tiempo de aparición de los anticuerpos es una respuesta personal, pueden ser detectados alrededor del séptimo día y en algunos casos varias semanas después del inicio de los síntomas. ${ }^{43-45}$ Algunos pacientes no desarrollan una respuesta inmune humoral; a esta incertidumbre se suma la relación poco clara entre la respuesta de anticuerpos y la mejoría clínica. No siempre se ha encontrado que la detección de anticuerpos y títulos más altos se correlacionen con la mejoría clínica. En pacientes con enfermedad leve, el COVID-19 puede resolverse antes de la seroconversión. ${ }^{43,44}$ Estas eventualidades son las principales dificultades de esta prueba. La durabilidad de los anticuerpos principalmente lgG contra el SARS-CoV-2 aún no se ha determinado. Se ha mencionado una persistencia de hasta 40 días desde el inicio de los síntomas; sin embargo, el tiempo real lo conoceremos pasada la pandemia. Aunque no se conocen reinfecciones, la detección de IgG no es garantía de inmunidad duradera. ${ }^{41-47}$ Las ventajas de este tipo de pruebas son su rapidez (5-15 minutos entre la toma de muestra y sus resultados); no requiere de técnica compleja para su procesamiento, ni de personal especializado; las desventajas son su limitada sensibilidad y especificidad (sensibilidad de $88.66 \%$ y especificidad de 90.63), con posibilidad de falsos negativos. ${ }^{3,48}$

5. Diagnóstico por estudios de imagen. La radiografía de tórax (CXR) tiene poco valor diagnóstico en las primeras etapas, llegando a ser normal en etapas intermedia o avanzada. Generalmente muestra infiltrados bilaterales, con progresión característica del síndrome de dificultad respiratoria aguda. ${ }^{2,6,49} \mathrm{La}$ tomografía simple de tórax (TAC) inició su aportación en la pandemia como complemento al diagnóstico y seguimiento de la enfermedad; sin embargo, gradualmente se volvió una herramienta fundamental en los centros clínicos donde hay escasez de las pruebas diagnósticas de laboratorio. Los 
principales hallazgos son las «opacidades en vidrio esmerilado», con una distribución periférica y subpleural. Lo más frecuente es encontrar involucrados múltiples lóbulos, principalmente los inferiores; estos hallazgos pueden encontrase incluso en pacientes asintomáticos sin evidencia clínica de la enfermedad.6,33,36 El engrosamiento septal, bronquiectasias, engrosamiento pleural y afectación subpleural son hallazgos menos comunes, principalmente en las etapas posteriores de la enfermedad. La etapa intermedia de la enfermedad muestra un aumento en el número y tamaño de infiltrados y opacidades multifocales consolidadas, engrosamiento septal y desarrollo de un patrón de pavimentación. La severidad de hallazgos visibles se presenta alrededor del día 10 después de iniciados los síntomas. ${ }^{4,36}$ Se ha reportado que la TAC tiene una sensibilidad de hasta el $98 \%$ frente al $71 \%$ de la PCR (60 a 89\%), incrementando con ello su importancia. ${ }^{27,50-55}$

Indicaciones de las pruebas diagnósticas. El principal método diagnóstico sigue siendo la PCR, pudiendo apoyarse con las IgM e IgG para conocer la fase de la enfermedad. Las pruebas existentes nunca deben utilizarse en forma aislada, de acuerdo con las recomendaciones de la OMS, salvo en el campo de la investigación. ${ }^{17,38}$ Por otro lado, la tomografía computarizada simple de tórax, a pesar de ser limitada para la identificación del virus, ha demostrado tener una baja tasa de omisión diagnóstica en casos de COVID-19, por lo que realizarla nos ayuda a tomar la decisión de tratar o aislar pacientes con base en sus hallazgos, especialmente en zonas que carecen de cualquier tipo de pruebas. La combinación de las pruebas con la TAC de tórax aumenta la sensibilidad y especificidad para el diagnóstico de COVID-19. ${ }^{27,48-64} \mathrm{La}$ recomendación es utilizar los métodos diagnósticos disponibles de manera conjunta, lo que dará una mayor certeza al diagnóstico de la enfermedad (Figura 2). 3,49,50

\section{IMPORTANCIA DE ESTRATIFICAR LOS PROCEDIMIENTOS QUIRÚRGICOS DURANTE LA PANDEMIA Y EL RETORNO A LAS ACTIVIDADES LABORALES}

Todos los pacientes que son operados durante la pandemia deben ser considerados como casos infectados o portadores con el riesgo de contribuir a la propagación del coronavirus dentro de las instalaciones. En todos los pacientes de cirugía urgente se deberán aplicar en forma estricta los protocolos de seguridad. Hay muchos pacientes asintomáticos que sin saberlo están eliminando virus y exponiendo a otros pacientes y al personal sanitario al riesgo de contagio. ${ }^{13,65-68}$ La contingencia obliga a revisar cuidadosamente todos los procedimientos electivos programados y no reflejan un presunto imperativo de cancelar toda cirugía electiva. La cancelación de cirugía electiva puede tener un impacto más dramático e inconmensurable en la salud de nuestras comunidades que la morbilidad y mortalidad infligida por la nueva enfermedad de coronavirus. ${ }^{13,65-77}$

Cada sistema de salud, hospital y cirujano debe revisar cuidadosamente todos los procedimientos programados con un plan para minimizar los riesgos, posponer o cancelar operaciones programadas hasta que hayamos pasado el punto de inflexión previsto en el gráfico de exposición y podamos estar seguros que nuestra infraestructura de atención médica pueda soportar un potencial rápido y abrumador aumento en las necesidades críticas de atención a los pacientes. ${ }^{13}$

A la luz de todos los supuestos e incertidumbres subyacentes, es imperativo diseñar e implementar algoritmos clínicamente relevantes y orientados a la seguridad de los pacientes para guiar la toma de decisiones en los procedimientos quirúrgicos. Se han propuesto varias guías para la estratificación de riesgo y recomendaciones de seguridad. ${ }^{13}$

1. Estratificación del riesgo basado en la puntuación de la escala COVID-19 y regreso a la actividad. Es una escala que puede ser de mucha utilidad en la selección de pacientes que pueden ser candidatos a cirugía durante la época de la pandemia. Los de menor puntuación tienen menor riesgo (Figura 3). 2,6,30,31

2. Estratificación del riesgo basado en la urgencia de atención quirúrgica (Figura 4). ${ }^{13}$ Esta escala toma en consideración el tiempo en que un paciente tiene que ser atendido de acuerdo con la severidad de su patología y la urgencia de la atención quirúrgica. 
- Emergente. Atención de los pacientes en un tiempo menor a una hora. Aunque los pacientes se encuentren en una condición crítica y en riesgo de muerte, el equipo quirúrgico deberá tomar el tiempo suficiente para la colocación del equipo de protección personal y planear el procedimiento.

- Urgente. Atención de los pacientes en un tiempo menor a 24 horas. Antes de la atención se le deberán solicitar exámenes y pruebas de detección de COVID; discutir con todo el equipo quirúrgico y prepararse para la cirugía. En los casos COVID positivo las precauciones deben ser extremas.

- Urgente electiva. Atención de los pacientes antes de dos semanas. Durante el tiempo de espera se deberán completar los estudios, preparar a los pacientes y planificar el procedimiento, tratando de disminuir el riesgo de contagio tanto para el paciente como para el personal.

- Electiva esencial. Atención de los pacientes en un tiempo de uno a tres meses. Los pacientes de este grupo tienen patologías que requieren de un tratamiento necesario, pero no urgente. No se puede retrasar la atención quirúrgica, hacerlo puede traer consecuencias severas para los pacientes. Se debe buscar la oportunidad cuando los servicios hospitalarios no estén sobresaturados por la pandemia.

- Electiva discrecional. Los pacientes podrán atenderse cuando la pandemia se haya controlado.

3. Estratificación de riesgo con base en el tipo de procedimiento y generación de aerosoles potencialmente con partículas virales y recomendaciones de seguridad. $^{78-80}$

Médicos y otros trabajadores de la salud que participan en exámenes y procedimientos en las regiones de la cabeza y el cuello, del tubo digestivo alto y de las vías respiratorias tienen un riesgo particularmente alto de contagio por la exposición y contaminación por aerosoles y gotas, reportes de Wuhan en China de 138 pacientes hospitalizados por COVID-19, 40 eran trabajadores de la salud. ${ }^{78-80}$

- Consulta de pacientes sin realizar procedimientos. La exploración física de cabeza y cuello que incluirá las mucosas se debe realizar manteniendo el nivel sugerido de precaución. El examen preferiblemente se debe realizar en una habitación separada de otros pacientes y sólo el personal necesario debe estar presente. El examen debe ser realizado por la persona más experimentada presente y podría ser una evaluación más centrada, basada en el juicio del examinador médico. ${ }^{78-80}$ En pacientes asintomáticos o con pruebas negativas para COVID-19, es recomendable cumplir con las recomendaciones sanitarias para las oficinas y consultorios. En los casos sospechosos o confirmados, tratar de diferir la consulta; en caso urgente, utilizar las recomendaciones para el manejo de pacientes con COVID-19.

- Procedimientos intervencionistas generadores de aerosoles. Incluyen, entre otros: anestesia general con intubación o extubación traqueal, endoscopia nasal, laríngea, broncoscopia o gastrointestinal. Cirugía de nariz o garganta, drenaje de absceso periamigdalino, traqueotomía, atención de traqueotomía. Instrumentación motorizada en cirugía de cabeza y cuello de la mucosa, posiblemente cirugía laparoscópica. Evaluación previa de ser posible y solicitar estudio para descartar COVID. Cuando se trata de pacientes con lesiones que pueden involucrar las superficies mucosas de la cabeza/cara, nariz y faringe, estos procedimientos deben considerarse generadores de aerosol y todas las guías institucionales apropiadas para la utilización de EPP se deben seguir. Los pacientes sospechosos, en estudio o con COVID confirmado, realizar los procedimientos en los quirófanos asignados para la atención de este tipo de pacientes; usar el equipo de protección personal estricto para todo 
el personal; filtros especiales en el aire acondicionado y en las mascarillas del personal; desinfección previa y posterior al procedimiento.

- Procedimientos de intervención no generadores de aerosoles. La cirugía de tejidos blandos expone liberación de sangre que puede tener un recuento viral. Cuando la sangre está en aerosol por el uso de dispositivos de energía, puede implicar un riesgo de contagio; sin embargo, hasta el momento no hay estudios que respalden esta teoría. Cuando se utilicen dispositivos de energía, se debe limitar su uso y el humo generado deberá ser absorbido por aspirados con filtros especiales. El equipo quirúrgico deberá utilizar mascarillas con filtros especiales y en el quirófano se deberán utilizar extractores de aire con filtros específicos para contener los virus.

4. Estratificación de riesgo basado en la edad, comorbilidades y tipo de trabajo. ${ }^{81}$

Aplica también para los cirujanos plásticos (Figura 5).

Las personas viejas con afecciones crónicas como diabetes, hipertensión arterial y obesidad se enfrentan a una mayor mortalidad de COVID-19. En una gran serie de casos en China, la tasa de mortalidad fue inferior al $0.5 \%$ entre las personas menores de 50 años de edad, $1.3 \%$ entre los 50 a 59 años y $3.6 \%$ entre 60 a 69 años. Las personas con diabetes tenían un riesgo de muerte tres veces mayor que el de la cohorte general. Estos datos sugieren que la tasa de letalidad puede aproximarse al 10\% para personas que tienen entre 60 y 69 años y además tienen diabetes, más de 20 veces mayor que entre las personas menores de 50 sin una afección crónica. El riesgo aumenta por la edad y por las enfermedades crónicas. Un hospital en España informó que el $11.6 \%$ de sus 6,800 empleados dieron positivo para el virus. ${ }^{2}$ Adicionalmente, altas tasas de infección han sido reportadas entre trabajadores que tienen contacto con el público.

- Riesgo alto. Personas mayores de 60 años de edad o jóvenes con enfer- medades crónicas en contacto con pacientes sospechosos o confirmados de COVID-19.

- Riesgo medio. Personas mayores en contacto con pacientes con un estatus de COVID incierto o gente joven sana en contacto con pacientes con COVID.

- Riesgo bajo. Personas mayores que trabajan desde casa, con estricto distanciamiento, o que evita atender pacientes sospechosos; gente joven que trabaja en casa o en contacto con pacientes con un estatus de COVID incierto.

\section{PREPARACIÓN PARA EL REGRESO A LOS PROCEDIMIENTOS DE LA ESPECIALIDAD}

La pandemia por COVID-19 aún no ha sido controlada, continuará siendo una amenaza durante un largo periodo de tiempo. El retorno a las actividades habituales podrá hacerse en forma segura una vez que el virus haya atenuado su virulencia o cuando aparezca una vacuna efectiva o tratamiento retroviral eficaz y seguro. El retorno a las actividades laborales aceptado por la OMS y la mayoría de los países se hace con el fin de reactivar la economía, aún con el riesgo de contagio. Varias asociaciones internacionales han elaborado guías prácticas con la finalidad de disminuir el riesgo de contagio de pacientes-personal, las cuales orientan a los cirujanos de cómo realizar procedimientos estéticos con un riesgo mínimo de contagio; también ayudan a preparar de forma segura los hospitales, clínicas, salas de tratamiento y consultorios. ${ }^{7-11,18,82}$

Las recomendaciones tienen dos objetivos: la seguridad de los pacientes y la prevención de la infección por COVID-19. Estas recomendaciones no son obligatorias, no establecen un nuevo estándar de atención y no sustituyen su criterio como médico y cirujano. Todos los cirujanos deben cumplir con las reglamentaciones proporcionadas por la Secretaría de Salud, hospitales, organismos reguladores locales, estatales y gobierno federal. La prevención implica reducir los procedimientos de generación de aerosoles, es decir, manipulación de la vía aérea, ventilación con mascarilla, aspiración abierta de la vía aérea, 
uso de dispositivos productores de energía. Para reducir riesgos tanto a los pacientes como al personal, la cirugía electiva no se deberá realizar en pacientes infectados con COVID-19. En los pacientes que necesiten cirugía urgente se deberá esperar los resultados del laboratorio que confirmen el diagnóstico de COVID-19. Los riesgos y beneficios de proceder o posponer deben ser sopesados. En los pacientes asintomáticos es recomendable realizar cuestionario para detección de pacientes sospechosos, TAC de tórax, PCR y pruebas inmunológicas 48 horas previas a la cirugía y mantener sano distanciamiento previo a la cirugía. Es importante identificar a los miembros del personal que puedan estar infectados con COVID-19, se deberán realizar pruebas diagnósticas a todo el personal. Antes de la cirugía, se debe realizar una reunión con todo el equipo quirúrgico para asegurarse de que todos entiendan el plan de anestesia y cirugía, esto permitirá un trabajo en equipo sin interrupciones y con mínimos riesgos. Es importante la capacitación y simulacros para preparar al personal sobre los cuidados para evitar el contagio. Los pacientes de cirugía estética deberán ser operados en los quirófanos libres de COVID y no exponerlos a un contagio. Los quirófanos de presión negativa son ideales para minimizar el riesgo de infección, normalmente están diseñados para tener circulación de aire a presión positiva. ${ }^{14,82-87}$

Consulte las recomendaciones de las autoridades sanitarias de su país para prácticas de apertura y reanudación de procedimientos electivos y para obtener recomendaciones adicionales. Procure estar actualizado respecto al comportamiento de la transmisión de COVID-19. Si los casos comienzan a aumentar, considere si su práctica deberá reducirse.

Recomendaciones para la consulta ${ }^{14,31,82-94}$

- Elabore un plan de trabajo con su personal.

- Comunicación con el paciente vía telefónica. Definir las expectativas apropiadas de la visita al consultorio y del procedimiento solicitado; establecer los riesgos asociados enfatizando el COVID-19. Enviar protocolos de actuación previa a la consulta presencial.
- Concertar las citas, incluyendo las consultas por videoconferencia.

- En la sala de espera, los pacientes deberán estar separados el uno del otro y evitar contacto innecesario con el personal de la clínica o consultorio.

- Modificación física del consultorio, áreas comunes y salas de tratamiento, adaptarlas a los nuevos requerimientos sanitarios.

- Limpieza y desinfección en cada área del consultorio, incluir dispositivos médicos, salas de tratamiento, artículos administrativos y áreas de recepción. Utilizar alcohol etílico al 70\%, hipoclorito de sodio al 0.5\% y luz ultravioleta (UV-C).

- Uso del equipo de protección personal (PPE) para personal y pacientes; usar dependiendo del riesgo.

- Preparar las salas de tratamiento para el ingreso del paciente en un ambiente reducido de riesgo de contagio por COVID-19.

- Enviar por medio electrónico y previo a la consulta presencial el consentimiento informado sobre los riesgos de los procedimientos a realizar y el riesgo de contagio por COVID. El paciente lo deberá entregar el día de la consulta presencial.

- Promover que el paciente acuda sólo a la consulta presencial o cirugía.

- Todos los pacientes que se presenten en el hospital deberán ser examinados usando un cuestionario estándar y dispositivos especiales como termómetro y oximetría. Los pacientes con sospecha deberán ser remitidos al especialista.

- Considerar que algunos tratamientos estéticos como la aplicación de toxina botulínica e inyecciones de relleno dérmico implican un riesgo por la cercanía a las mucosas respiratorias y digestivas, así como el uso de láser no invasivo y otros dispositivos emisores de energía similares, pueden producir aerosoles. En ambos casos se debe tener especial cuidado en el uso del EPP.

- Planificar las medidas de acuerdo a cada fase de la pandemia.

- Es recomendable que el pago de honorarios se haga de forma electrónica.

- Revisar las evidencias actuales y realizar los cambios a las guías. 
Recomendaciones para la aneste$\operatorname{sia}^{9,12-14,31,65-79,82-94}$

- Utilizar preferentemente anestesia regional.

- Evitar la intubación con el paciente despierto, la tos del paciente, como la anestesia local atomizada, pueden conducir a la aspersión del virus. ${ }^{25,95}$

- Usar filtros adecuados en las máquinas de anestesia y en los extractores de aire.

- Si se elige anestesia regional, colocar al paciente una mascarilla tipo N95; se puede administrar oxígeno suplementario por vía nasal debajo de la mascarilla.

- Reducir la necesidad de desconexiones de circuitos, se deben adjuntar extensiones antes de comenzar el procedimiento.

- Asegurar la vía aérea con un tubo endotraqueal; no se recomienda la cánula supraglótica (laríngea).

- Se recomienda el uso de videolaringoscopio porque la careta o gafas se pueden empañar y dificultar la visión durante la laringoscopia.

- La secuencia de inducción anestésica deberá ser rápida, para reducir la necesidad de ventilación con bolsa y mascarilla.

- Anestesia profunda y el bloqueo neuromuscular son importantes antes de intentar la intubación.

- El anestesiólogo experimentado debe intubar. ${ }^{25}$

- Se debe usar succión traqueal cerrada en línea en lugar de succión abierta. ${ }^{95}$

- Minimizar las desconexiones del circuito es ideal, pero si esto es inevitable, asegure una presión positiva si la ventilación ha cesado, gire la limitación de presión ajustable válvula a cero y considere sujetar el tubo endotraqueal antes de la desconexión. Esta técnica también se puede usar antes de cambiar a un paciente de cuidados intensivos de transportar el ventilador a la máquina de anestesia. El paciente debe estar preoxigenado y la duración de la desconexión debe mantenerse al mínimo para evitar exacerbar hipoxia en pacientes críticos con COVID-19.25

- Aplicar antieméticos para reducir las arcadas postoperatorias y la formación de aerosoles.
- Usar un catéter de succión rígido para reducir la posibilidad de contaminar el entorno con el catéter de succión suave y flexible.

- Evitar las máscaras Venturi, ya que pueden asperjar el virus. ${ }^{25}$

- Se debe preferir el equipo desechable para la vía aérea.

- El paciente se debe recuperar dentro del quirófano mismo.

Recomendaciones para los procedimientos quirúrgicos $9,12-14,31,65-79,82-94$

- La cirugía electiva se pospone si el paciente es sospechoso o viajó a las zonas de mayor contagio.

- Comprobar la desinfección del área de trabajo y de todos los aparatos que se utilicen en quirófano.

- Se deben asegurar estrategias de planificación para realizar cirugías urgentes durante la pandemia.

- Deberá ser cuidadoso en procedimientos laparoscópicos y abiertos con dispositivos de aspiración, cauterización e insuflación. La presión neumoperitoneal debe ser baja. La potencia del electrocauterio debe ser lo más baja posible para minimizar las dispersiones de partículas contaminantes. ${ }^{23}$

- Realizar una esterilización rigurosa de la habitación y materiales para garantizar la seguridad del próximo paciente y también del equipo de atención. ${ }^{22}$ Controlar o cancelar las visitas de los pacientes para minimizar el tráfico de personas y materiales, garantizando la disminución de la exposición a otras personas que pueden ser portadores asintomáticos. $^{22}$

- Todo el personal debe estar específicamente capacitado para el uso del equipo de protección personal (EPP). Los médicos con barba deben tener especial atención al ajuste de la máscara para garantizar una protección adecuada. ${ }^{1,16}$

- Usar viseras o gafas para proteger los ojos de la exposición potencial de partículas virales. ${ }^{1,21}$

- Usar dobles guantes. ${ }^{96}$

- Cubrir la boca y nariz de ser posible y colocar una barrera entre la cara y la región a operar. ${ }^{96}$ 
- Operar pacientes de bajo riesgo; la cirugía no debe rebasar 3.5 horas. ${ }^{96}$

\section{CONCLUSIONES}

La pandemia por COVID-19 continuará por un largo tiempo; el retorno a las actividades habituales se está realizando con fines económicos y no porque la pandemia se haya controlado. Para disminuir el riesgo de contagio es necesario tener conocimiento de la enfermedad y utilizar las guías emitidas por diferentes organizaciones y las recomendaciones indicadas por las autoridades sanitarias.

Es necesario realizar las pruebas diagnósticas tanto en los pacientes como en el personal. En el paciente para garantizar que no sean enfermos asintomáticos que puedan contagiar a otras personas o que la enfermedad no se complique durante el procedimiento estético. En el personal para detectar los casos positivos, los enfermos activos y los recuperados y de esa forma establecer una estrategia para emplear el personal de menor riesgo.

La pandemia nos está obligando a cambios importantes en la atención de nuestros pacientes. Nuestros consultorios, salas de tratamiento y quirófanos deberán implementar cuidados sanitarios y estar libres de COVID por el bien de nuestros pacientes y del nuestro.

Es importante mantenerse informado del desarrollo de la pandemia y de los avances de tratamiento que van surgiendo, así como revisar las evidencias actuales y realizar los cambios a las guías.

\section{REFERENCIAS}

1. Dawood A. Mutated COVID-19 may foretell a great risk for mankind in the future. Microbes New Infect 2020; 35: 100673.

2. Zheng SQ et al. Recommendations and guidance for providing pharmaceutical care services during COVID-19 pandemic: A China perspective. Res Social Adm Pharm 2020. PMID: 32249102.

3. World Health Organization website. Pneumonia of unknown cause. Accessed February 13, 2020. [Google Scholar] Available in: China.www.who.int/ csr/don/05-january-2020-pneumonia-of-unkowncause-china/en/

4. World Health Organization. WHO Director-General's Remarks at the Media Briefing on 2019-nCoV on 11 February 2020. 2020. Available in: https://www. who.int/dg/speeches/ detail/who-director-general-s- remarks-at-the-media-briefing-on-2019-ncov-on-11february-2020.

5. Zhang L, Liu Y. Potential interventions for novel coronavirus in China: a systemic review. J Med Virol 2020. https://doi.org/10. 1002/jmv.25707.Tanu Singal.

6. Singhal T. A review of coronavirus disease-2019 (COVID-19). Indian J Ped 2020; 87 (4): 281-286.

7. Sohrabi $\mathrm{C}$ et al. World Health Organization declares global emergency: a review of the 2019 novel coronavirus (COVID-19). Int J Surg 2020; 76: 71-76.

8. Brindle M, Gawande A. Managing COVID-19 in surgical systems. Ann Surg 2020. doi: 10.1097/ SLA.0000000000003923.

9. Coccolini F, Perrone G, Chiarugi M, Di Marzo F. Surgery in COVID-19 patients: operational directives. World J Emerg Surg 2020; 15 (1): 25.

10. Kirkcaldy RD, King BA, Brooks JT. COVID-19 e inmunidad posinfección. Evidencia limitada, muchas preguntas restantes JAMA 2020. doi: 10.1001 / jama.2020.7869.

11. Gandhi M, Yokoe DS, Havlir DV. Asymptomatic transmission, the Achilles' heel of current strategies to control COVID-19. N Engl J Med 2020; 382: 21582160. doi: 10.1056 / NEJMe2009758.

12. Saúde MD. Cirurgias seguras salvam vidas. Rio de Janeiro: Organização Pan-Americana da Saúde, Ministério da Saúde, Agência Nacional de Vigilância Sanitária; 2009. Available in: http://bvsms.saude.gov. br/bvs/publicacoes/seguranca_paciente_cirurgias_ seguras_guia.pdf.

13. Stahel PF. How to risk-stratify elective surgery during the COVID-19 pandemic? Patient Saf Surg 2020; 14: 8.

14. Wong J, Goh QY, Tan Z et al. Preparing for a COVID-19 pandemic: a review of operating room outbreak response measures in a large tertiary hospital in Singapore. Can J Anaesth 2020; 67 (6): 732-745. doi: 10.1007/s12630-020-01620-9.

15. World Health Organization. Q\&A on coronavirus (COVID-19). [accessed March 2020] Available in: https://www.who.int/news-room/q-a-detail/q-acoronaviruses

16. European Centre for Disease Prevention and Control. COVID-19. [accessed March 2020] Available in: https://www.ecdc.europa.eu/en/novelcoronaviruschina

17. Poutanen SM, McGeer AJ. Transmission and control of SARS. Curr Infect Dis Rep 2004; 6: 220-227.

18. Yeo C, Kaushal S, Yeo D. Enteric involvement of coronaviruses: is faecal-oral transmission of SARSCoV-2 possible? Lancet Gastroenterol Hepatol 2020. doi: https://doi.org/10.1016/S24681253(20)30048-0.

19. Centers for Disease Control and Prevention. How COVID-19 spreads. [accessed March 2020] Available in: https://www.cdc.gov/coronavirus/2019-ncov/about/ transmission.html

20. Scales DC, Green K, Chan AK et al. Illness in intensive care staff after brief exposure to severe acute respiratory syndrome. Emerg Infect Dis 2003; 9: 12051210.

21. Loeb M, McGeer A, Henry B et al. SARS among critical care nurses, Toronto. Emerg Infect Dis 2004; 10 (2): 251-255. 
22. Riou J, Althaus CL. Pattern of early human-to-human transmission of Wuhan 2019 novel coronavirus (2019nCoV), December 2019 to January 2020. Euro Surveill 2020; 25 (4): 2000058. doi: 10.2807/1560-7917. ES.2020.25.4.2000058.

23. Li Q, Guan X, Wu P et al. Early transmission dynamics in Wuhan, China, of novel coronavirus-infected pneumonia. N Engl J Med 2020. doi: https://doi. org/10.1056/nejmoa2001316.

24. Laboratory testing for 2019 novel coronavirus (2019$\mathrm{nCoV}$ ) in suspected human cases. Interim guidance. 2020. [Consulted 31 March 2020] Available in: https:// www.who.int/publications-detail/laboratory-testingfor-2019-novel-coronavirus-in-suspected-humancases-20200117

25. Pan Y, Zhang D, Yang P, Poon LLM, Wang Q. Viral load of SARS-CoV-2 in clinical samples. Lancet Infect Dis 2020; 20 (4): 411-412.

26. Loeffelholz MJ, Tang YW. Laboratory diagnosis of emerging human coronavirus infections - the state of the art. Emerg Microb Infect 2020; 9 (1): 747-756.

27. World Health Organization. Advice on the use of point-of-care immunodiagnostic tests for COVID-19. Scientific brief. 2020. Available in: https://www. who.int/docs/default-source/coronaviruse/sb2020-1-poc-immunodiagnostics-2020-04-08-e. pdf?sfvrsn $=4$ c26ac39 2

28. Adhikari SP, Meng S, Wu YJ et al. Epidemiology, causes, clinical manifestation and diagnosis, prevention and control of coronavirus disease (COVID-19) during the early outbreak period: a scoping review. Infect Dis Poverty 2020; 9 (1): 29.

29. OMS desaconseja uso de test rápidos. Disponible en: https://www.infosalus.com/asistencia/noticia-omsdesaconseja-uso-test-rapidos-anticuerpos-covid-19

30. COVID19/cuestionarioenlínea/cdmx.gob.mx.

31. Dover JS, Moran ML, Figueroa JF et al. A path to resume aesthetic care executive summary of project AesCert ${ }^{\mathrm{TM}}$ guidance supplement: practical considerations for aesthetic medicine professionals supporting clinic preparedness in response to the SARS-CoV-2 outbreak. Facial Plast Surg Aesthet Med 2020. doi:10.1089/ abc.2020.0239.

32. Huang C, Wang Y, Li X, Ren L, Zhao J, Hu Y et al. Clinical features of patients infected with 2019 novel coronavirus in Wuhan, China. Lancet 2020; 395: 497 506. https://doi.org/10.1016/S0140-6736(20)30183-5.

33. CDC. 2019 Novel coronavirus. Wuhan, China. 2020. [Accessed 1 Feb 2020] Available in: https://www.cdc. gov/coronavirus/2019-nCoV/summary.html

34. Alhazzani W, Hylander M, Arabi YM et al. Surviving sepsis campaign: Guidelines of management of critically III adults with Coronavirus Disease 2019 (Covid-19). Crit Care Med J 2020; 28 (6): 440-469.

35. Corman VM, Landt O, Kaiser $\mathrm{M}$ et al. Detection of 2019 novel coronavirus (2019-nCoV) by real-time RT-PCR. Euro Surveill 2020; 25: 2000045.

36. Chu DKW, Pan Y, Cheng SMS et al. Molecular diagnosis of a novel coronavirus (2019-nCoV) causing an outbreak of pneumonia. Clin Chem 2020; 66: 549-555.

37. Bao L, Deng W, Gao H et al. Falta de reinfección en macacos Rhesus infectados con SARS-CoV-2. bioRxiv. 2020. doi: 10.1101 / 2020.03.13.00-226.
38. Cavanaugh D. Coronavirus y torovirus. En: Zuckerman AJ, Banatvala JE, Pattinson JR, Griffiths PD, Schoub BD, Ed. Principios y práctica de la virología clínica. 5a ed. John Wiley \& Sons Ltd; 2004. pp. 379-397. doi: 10.1002/0470020970.ch10.

39. Xing Y, Mo P, Xiao Y, Zhao O, Zhang Y, Wang F. Vigilancia post-alta y la detección de virus positivo en dos de personal médico se recuperaron de la enfermedad coronavirus 2019 (COVID-19), China, enero-febrero de 2020. Euro Surveill 2020; 25 (10). doi: 10.2807/1560-7917.ES.2020.25.10.2000191

40. Xiao AT, Tong YX, Zhang S. Falso negativo de RTPCR y conversión prolongada de ácido nucleico en COVID-19: en lugar de recurrencia. J Med Virol 2020. doi: 10.1002/jmv.25855

41. Young BE, Ong SWX, Kalimuddin S et al; Equipo de investigación de brotes de coronavirus novedoso de Singapur 2019. Características epidemiológicas y curso clínico de pacientes con SARS-CoV-2 en Singapur. JAMA 2020; 232 (15): 1488-1494. doi: 10.1001/ jama.2020.3204

42. Zhao J, Yuan Q, Wang $\mathrm{H}$ et al. Las respuestas de anticuerpos a SARS-CoV-2 en pacientes de la enfermedad de coronavirus novedoso 2019. Clin Infect Dis 2020. doi: 10.1093/cid/ciaa344

43. Wölfel R, Corman VM, Guggemos W et al. Evaluación virológica de pacientes hospitalizados con COVID-2019. Naturaleza 2020. doi: 10.1038/ s41586-020-2196-x

44. To KK, Tsang OT, Leung WS et al. Perfiles temporales de carga viral en muestras de saliva orofaríngea posterior y respuestas de anticuerpos en suero durante la infección por SARS-CoV-2: un estudio observacional de cohorte. Lancet Infect Dis 2020; 20 (5): 565-574. doi: 10.1016/S1473-3099(20)30196-1

45. Wu LP, Wang NC, Chang YH et al. Duración de las respuestas de anticuerpos después del síndrome respiratorio agudo severo. Emerg Infect Dis 2007; 13 (10): 1562-1564. doi: 10.3201/eid1310.070576

46. Payne DC, Iblan I, Rha B et al. Persistencia de anticuerpos contra el coronavirus del síndrome respiratorio de Oriente Medio. Emerg Infect Dis 2016; 22 (10): 1824-1826. doi: 10.3201/ eid2210.160706

47. Zhang W, Du RH, Li B et al. Molecular and serological investigation of 2019-nCoV infected patients: implication of multiple shedding routes. Emerg Microbes Infect 2020; 9 (1): 386-389. doi: 10.1080/22221751.2020.1729071.

48. Sociedad Española de Inmunología. Utilidad de la determinación de anticuerpos anti SARSCoV-2. Propuesta de implementación como prueba diagnóstica, pronóstica y de desarrollo de inmunidad protectora. 2020 [Consultado 5 abril 2020].

49. Ai T, Yang Z, Hou H et al. Correlation of chest CT and RT-PCR testing in coronavirus disease 2019 (COVID-19) in China: a report of 1014 cases. Radiology 2020 [online ahead of print].

50. Fang Y, Zhang H, Xie J et al. Sensitivity of chest CT for COVID-19: comparison to RT-PCR. Radiology 2020; 200432.

51. Hani C, Trieu NH, Saab I et.al. Covid-19 Pneumonia: a review typical CT findings and differential diagnosis. 
Diagnostic and Interventional Imaging 2020; 101: 263268.

52. Pan F, Ye T, Sun P, Gui S, Liang B, Li L et al. Time course of lung changes on chest CT during recovery from 2019 novel coronavirus (COVID-19) pneumonia. Radiology 2020; 200370. doi: 10.1148/radiol.2020200370.

53. Shi H, Han X, Cao Y, Alwalid O, Zheng C. CT screening for early diagnosis of SARS-CoV-2 infection-authors' reply. Lancet Infect Dis 2020. doi: 10.1016/S14733099(20)30247-4.

54. Salehi S, Abedi A, Balakrishnan S, Gholamrezanezhad A. Coronavirus disease 2019 (COVID-19): a systematic review of imaging findings in 919 patients. AJR Am J Roentgenol 2020. http://dx.doi.org/10.2214/ AJR.20.23034.

55. Yuan M, Yin W, Tao Z, Tan W, Hu Y. Association of radiologic findings with mortality of patients infected with 2019 novel coronavirus in Wuhan, China. PLoS One 2020; 15: e0230548.

56. Xie X, Zhong Z, Zhao W, Zheng C, Wang F, Liu J. Chest CT for typical 2019-nCoV pneumonia: relationship to negative RT-PC Testing. Radiology 2020; 200343 doi: 10.1148/radiol.2020200343.

57. Xu X, Yu C, Qu J, Zhang L, Jiang S, Huang D et al. Imaging and clinical features of patients with 2019 novel coronavirus SARS-CoV-2. Eur J Nucl Med Mol Imaging 2020; 47: 1275-1280.

58. Kanne JP. Chest CT findings in 2019 novel coronavirus (2019-nCoV) infections from Wuhan, China: key points for the radiologist. Radiology 2020; 295 (1): 16-17.

59. Feng H, Liu Y, Lv M, Zhong J. A case report of COVID-19 with false negative RT-PCR test: necessity of chest CT. Jpn J Radiol 2020. doi:1 0.1007/s11604020-00967-9

60. American College of Radiology. ACR recommendations for the use of chest radiography and computed tomography (CT) for suspected COVID-19 infection. 2020. Available in: https://www.acr.org/ Advocacy-and-Economics/ACR-Position- Statements/ Recommendations-for-Chest-Radiography-and-CTfor-Suspected-COVID19- Infection

61. Li Y, Xia L. Coronavirus disease 2019 (COVID-19): role of chest CT in diagnosis and management. AJR 2020; 214: 1280-1286.

62. Winichakoon $\mathrm{P}$, Chaiwarith $\mathrm{R}$, Liwsrisakun $\mathrm{C}$ et al. Negative nasopharyngeal and oropharyngeal swab does not rule out COVID-19. J Clin Microbiol 2020.

63. Guan W, Ni Z, Hu Y et al. Clinical characteristics of 2019 novel coronavirus infection in China. 2020. doi: https://doi.org/10.1101/2020.02.06.20020974.

64. Rubin GD, Ryerson CJ, Haramati LB et al. The role of chest imaging in patient management during the COVID-19 pandemic: a multinational consensus statement from the Fleischner Society. Chest 2020. doi: 10.1016/j.chest.2020.04.003.

65. McKay B, Calfas J, Ansari T. Coronavirus declared pandemic by World Health Organization. Wall St J 2020.

66. Commins J. Surgeon general urges providers to consider stopping all elective surgeries - hospitals push back. Health Leaders 2020.

67. Evans M, Wilde MA. Hospitals push off surgeries to make room for coronavirus patients. Wall St J 2020.
68. Martines J. UPMC shuns health experts' calls to cancel elective surgeries. Pittsburgh Tribune-Review 2020.

69. Sathya C. Your elective surgery will be canceled. It's for everyone's good. The Washington Post 2020.

70. American Hospital Association (AHA), the Association of American Medical Colleges (AAMC), the Children's Hospital Association (CHA), and the Federation of American Hospitals (FAH). Open letter to Vice Admiral Jerome M. Adams, MD, United States Surgeon General. March 15, 2020.

71. American College of Surgeons (ACS). COVID-19 update: guidance for triage.

72. Zhang S. What it really means to cancel elective surgeries: to make room for coronavirus patients, hospitals are delaying procedures that would make major differences in people's lives. The Atlantic 2020.

73. Yu GY, Lou Z, Zhang W. Several suggestion of operation for colorectal cancer under the outbreak of corona virus disease 19 in China. Zhonghua Wei Chang Wai Ke Za Zhi 2020 [Feb 19, Epub ahead of print].

74. Weise K, Baker M, Bogel-Burroughs N. The coronavirus is forcing hospitals to cancel surgeries. The New York Times 2020.

75. Beasley D. United States hospitals and patients cancel elective surgery as coronavirus spreads. The New York Times 2020

76. Ren SY, Gao RD, Chen YL. Fear can be more harmful than the severe acute respiratory coronavirus disease 2019 epidemic. World J Clin Cases 2020; 8: 652-657.

77. Awad ME, Rumley JCL, Vazquez JA, Devine JG. Perioperative considerations in urgent surgical care of suspected and confirmed COVID-19 orthopaedic patients: operating room protocols and recommendations in the current COVID-19 pandemic. J Am Acad Orthop Surg 2020; 28: 451-463. doi: 10.5435/JAAOS-D-20-00227.

78. Edwards SP, Kasten S, Nelson C, Elner V, McKean E. Maxillofacial trauma management during COVID-19: multidisciplinary recommendations. Facial Plast Surg Aesthet Med 2020; 22 (3): 157-159.

79. Givi B, Schiff BA, Chinn SB etal. Safety recommendations for evaluation and surgery of the head and neck during the COVID-19 pandemic. JAMA Otolaryngol Head Neck Surg 2020. doi: 10.1001/jamaoto.2020.0780.

80. Wang D, Hu B, Hu C et al. Clinical characteristics of 138 hospitalized patients with 2019 novel coronavirusinfected pneumonia in Wuhan, China. JAMA 2020. doi: 10.1001/jama.2020.1585.

81. Larochelle MR. "Is it safe for me to go to work?" Risk stratification for workers during the Covid-19 pandemic. N England Med 2020. doi: 0.1056/ NEJMp2013413.

82. Ti LK, Ang LS, Foong TW. Ng BSW what we do when a COVID-19 patient needs an operation: operating room preparation and guidance. Can J Anaesth 2020; 67 (6): 756-758. doi: 10.1007/s12630-020-01617-4

83. Surgeons ACo. Covid-19 recommendations for management of elective surgical procedures. Available in: https:/www.facs.org/about-acs/covid-19/ information-for-surgeons.

84. Aminian A, Safari S, Razeghian-Jahromi A, Ghorbani M, Delaney CP. Covid-19 outbreak and surgical practice: 
unexpected fatality in perioperative period. Ann Surg 2020. doi: 10.1097/SLA.0000000000003925.

85. Correia MITD, Ramos RF, Bahten LCV. The surgeons and the COVID-19 pandemic. Rev Col Bras Cir. 2020; 47: e20202536.

86. Centers for Disease Control and Prevention. Coronavirus Disease 2019 (COVID-19) situation summary. Available in: https://www.cdc.gov/ coronavirus/2019-ncov/summary.html

87. Plan de acción en la atención en consulta en el periodo posterior a la crisis de COVID-19 en cirugía plástica, estética y reparadora. Sociedad Española de Cirugía Plástica 2020.

88. Diaz A, Sarac BA, Schoenbrunner AR, Janis JE, Pawlik TM. Elective surgery in the time of COVID-19. Am J Surg 2020; 219 (6): 900-902.

89. Poulose BK, Phieffer LS, Mayerson J, Like D, Forrest LA, Rahmanian A et al. Responsible return to essential and non-essential surgery during the COVID-19 pandemic. J Gastrointest Surg 2020; 4: 1-3.

90. Dexter F, Parra MC, Brown JR, Loftus RW. Perioperative COVID-19 defense: an evidence-based approach for optimization of infection control and operating room management. Anesth Analg 2020; 131 (1): 37-42.

91. Andersen BM, Bånrud H, Bøe E, Bjordal O, Drangsholt F. Comparison of UV-C light and chemicals for disinfection of surfaces in hospital isolation units. Infect Control Hosp Epidemiol 2006; 27: 729-734.

92. Manual de procedimientos para la atención en cirugía plástica en consulta, clínicas de cirugía ambulatoria y quirófanos, durante la contingencia por SARS-COV2 (COV-19) Asociación Mexicana de Cirugía Plástica y Reconstructiva. 5 de junio de 2020.
93. Claytor B, Fernau J, Fiala T, Furnas H, Hamori C, Lund $\mathrm{H}$ et al. Recommendations from: the aesthetic society COVID-19 safety task force: reopening office and resuming elective procedures. 2020. Available in: https://www.cdc.gov/coronavirus/2019-ncov/hcp/ ppe-strategy/face- masks.html

94. D'Amico RS, Baum G, Serulle Y, Silva D, Smith $M L$, Wallack RA et al. A roadmap to reopening a neurosurgical practice in the age of COVID-19. World Neurosurg 2020; 139: 289-293.

95. Li T, Wei C, Li W, Hongwei F, Shi J. Beijing Union Medical College Hospital on "pneumonia of novel coronavirus infection" diagnosis and treatment proposal (V2.0). Med J Peking Union Med Coll Hosp 2020. [Accessed 2 Feb 2020] Available in: http://kns. cnki.net/kcms/detail/11.5882.r.20200130.1430.002. html

96. ISAPS Recommendations for the resumption of Esthetic Surgery. Available in: https://www.youtube.com/watc $\mathrm{h} ? \mathrm{v}=\mathrm{iZYQbaNcUSw \& feature=youtu}$.be

97. Rothe C, Schunk M, Sothmann P et al. Transmission of 2019nCoV infection from an asymptomatic contact in Germany. N Engl J Med 2020. doi: https://doi. org/10.1056/NEJMc2001 468.

\section{Correspondencia:}

\section{Dr. Jesús A Cuenca-Pardo}

Jerez Núm. 51, Col. Mixcoac, 03920, Alcaldía Benito Juárez, Ciudad de México, México. E-mail: jcuenca001@gmail.com 


\section{RECOMENDACIONES DE SEGURIDAD}

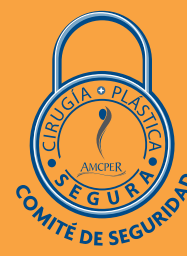

Parte I

\section{Figura 1}

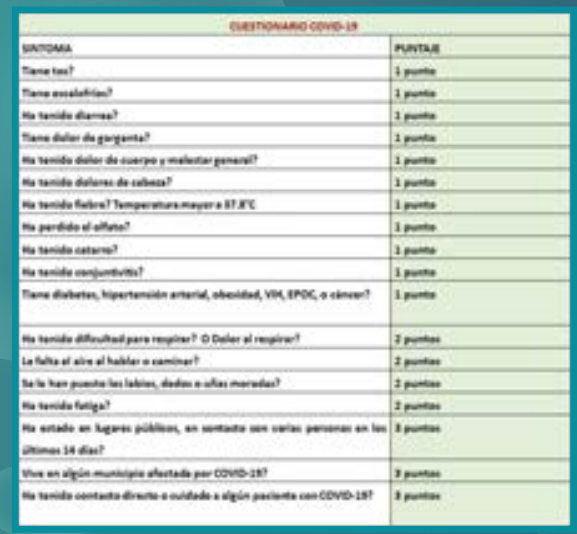

\section{Figura 2}

\section{Indicaciones}

\section{de pruebas diagnósticas.}

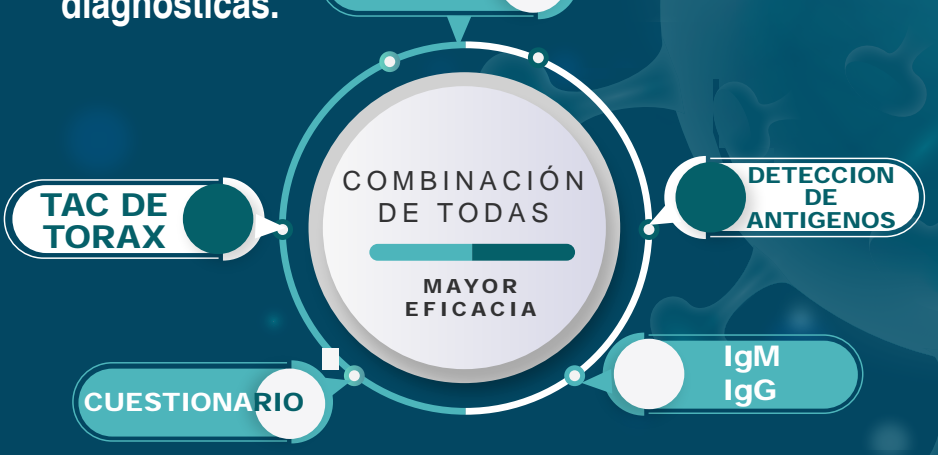

\section{Figura 3}

\begin{tabular}{|c|c|c|c|c|c|}
\hline \multicolumn{6}{|c|}{ RESULTADO DE LAS FRUEBAS DLAGNOSTCAS Y SU SICNIHICADO } \\
\hline PCR & $\operatorname{IEM}$ & lec & TaCtórax & Infección & Inmunidad \\
\hline nezativo & Nezativo & Negative & negativo- & No & No \\
\hline negative & positivo & negative & negativo & Falso positivo & no \\
\hline positivo & Nefativo & Negnivor. & negative- & Si e falso + & no \\
\hline negativo & Nezativo & Negativer & Positiver & $\begin{array}{l}\text { Sospechoso; alta } \\
\text { pesiltidad }\end{array}$ & No \\
\hline positivo & Neqativo & Negativer & positive. & si & no \\
\hline positivo & positivo & Negativor & positiver. & Inteoción acuda & Sin inmunidad \\
\hline positivo & positive & positivo- & positive. & $\begin{array}{l}\text { Infeseión sub: } \\
\text { aguda }\end{array}$ & con inmunidad \\
\hline $\begin{array}{l}\text { Poultivo o } \\
\text { negativo }\end{array}$ & netative & pesitivo- & positiver & $\begin{array}{l}\text { Infezeión fase } \\
\text { final }\end{array}$ & con inmunidad \\
\hline negativo & megative & pesitivo- & $\begin{array}{l}\text { Positive; } \\
\text { cambios tiplicos }\end{array}$ & $\begin{array}{l}\text { En reselueióno } \\
\text { curado }\end{array}$ & con inmunidad \\
\hline
\end{tabular}
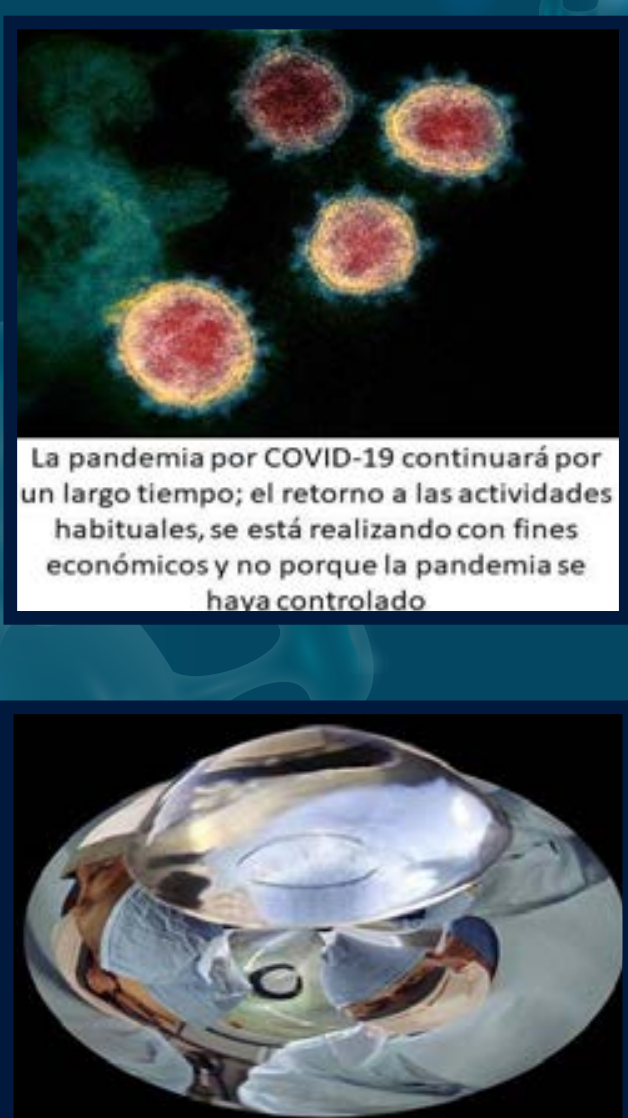

Los procedimientos estéticos pueden realizarse con un mínimo riesgo, sí tenemos conocimiento de la enfermedad y utilizamos guias con recomendaciones de seguridad

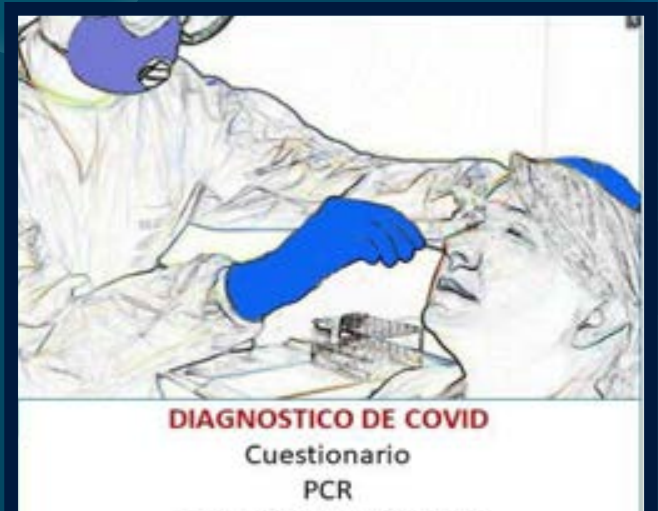

Detección de antígenos Determinación anticuerpos IgM e IgG Tomografía simple de tórax 
